\title{
Robust creation of entanglement between remote memory qubits
}

\author{
Bo Zhao, ${ }^{1}$ Zeng-Bing Chen, ${ }^{2}$ Yu-Ao Chen, ${ }^{1}$ Jörg Schmiedmayer,${ }^{1,3}$ and Jian-Wei Pan ${ }^{1,2}$ \\ ${ }^{1}$ Physikalisches Institut, Universität Heidelberg, Philosophenweg 12, D-69120 Heidelberg, Germany \\ ${ }^{2}$ Hefei National Laboratory for Physical Sciences at Microscale and Department of Modern Physics, \\ University of Science and Technology of China, Hefei, Anhui 230026, China \\ ${ }^{3}$ Atominstitut der Österreichischen Universitäten, TU-Wien, A-1020 Vienna, Austria
}

\begin{abstract}
In this Letter we propose a robust quantum repeater architecture building on the original DLCZ protocol [L.M. Duan et al., Nature 414, 413 (2001)]. The architecture is based on two-photon Hong-Ou-Mandel-type interference which relaxes the long distance stability requirements by about 7 orders of magnitude, from sub wavelength for the single photon interference required by DLCZ to the coherence length of the photons. Our proposal provides an exciting possibility for robust and realistic long distance quantum communication.
\end{abstract}

PACS numbers: 03.67.Hk,03.67.Pp,42.50.-p

Quantum communication holds the promise in achieving long-distance secure message transmission by exploiting quantum entanglement between remote locations [1, 2]. For long-distance quantum communication one must realize quantum network via quantum repeater protocol [3], a combination of entanglement swapping, entanglement purification and quantum memory. In a seminal paper [4], Duan et al. (DLCZ) proposed a promising implementation of the quantum repeater with atomic ensembles as local memory qubits and linear optics. In the effort of realizing DLCZ protocol, significant progress has been achieved in recent years [5, 6, 7].

However, entanglement generation and entanglement swapping in DLCZ protocol depend on Mach-Zehndertype interference. The relative phase between two remote entangled pairs is sensitive to path length instabilities, which has to be kept constant within a fraction of photon's wavelength. Moreover, entanglement generation and entanglement swapping are probabilistic. If connecting neighboring entangled pairs doesn't succeed after performing entanglement swapping, one has to repeat all previous procedures to reconstruct the entangled pairs. This means the path length fluctuation must be stabilized until the desired remote entangled pairs are successfully generated. A particular analysis shows that, to maintain path length phase instabilities at the level of $\lambda / 10$ ( $\lambda$ :wavelength; typically $\lambda \sim 1 \mu m$ for photons generated from atomic ensembles) requires the fine control of timing jitter at a sub-femto second level over a timescale of a few tens of seconds, no matter whether entanglement generation is performed locally or remotely. It is extremely difficult for current technology to meet this demanding requirement, since the lowest reported jitter is about a few tens of femto-seconds for transferring a timing signal over kilometer-scale distances for averaging times of $\geq 1 \mathrm{~s}$ [8].

As is well known, the two-photon Hong-Ou-Mandeltype interference is insensitive to phase instability. The path length fluctuations should be kept on the length scale within a fraction of photon's coherence length (say,
$1 / 10$ of the coherence length, which is about $3 \mathrm{~m}$ for photons generated from atomic ensembles [9]). Therefore the robustness is improved about 7 orders of magnitude higher in comparison with the single-photon MachZehnder-type interference in DLCZ protocol. The interference of two photons from independent atomic ensembles has been reported recently [10]. This type of two-photon interference has been widely used in quantum communication and quantum computation 11, 12.

To exploit the advantage of two-photon interference, it is natural to extend the DLCZ protocol by polarization encoding a memory qubit with two atomic ensembles [13], and entangling two memory qubits at neighboring sites via a two-photon Bell-state measurement (BSM). Unfortunately, the BSM won't create the desired entangled state, but a complex superposition state with spurious contributions from second-order excitations, which preclude further entanglement manipulation (see details below).

In this Letter, we explore this problem and find that by appropriate designing the BSM, the spurious contributions from second-order excitations can be automatically eliminated when entanglement swapping is performed. Motivated by this advance we propose a robust quantum repeater architecture with atomic ensembles and linear optics. This scheme makes use of the two-photon HongOu-Mandel-type interference, which is about 7 orders of magnitude more insensitive to path length phase instability than DLCZ scheme, and thus enables a robust and feasible implementation of long-distance quantum communication.

The basic element of the quantum repeater is a pencil shaped atomic sample of $N$ atoms with $\Lambda$ level structure (see inset in Fig. 1). The write laser pulse induces a spontaneous Raman process, which prepares the forwardscattered Stokes mode and collective atomic state into a two mode squeezed state. The light-atom system can be described as [4]

$$
|\psi\rangle=\left|0_{a} 0_{s}\right\rangle+\sqrt{\chi} S^{\dagger} a^{\dagger}\left|0_{a} 0_{s}\right\rangle+\chi \frac{\left(S^{\dagger} a^{\dagger}\right)^{2}}{2}\left|0_{a} 0_{s}\right\rangle
$$




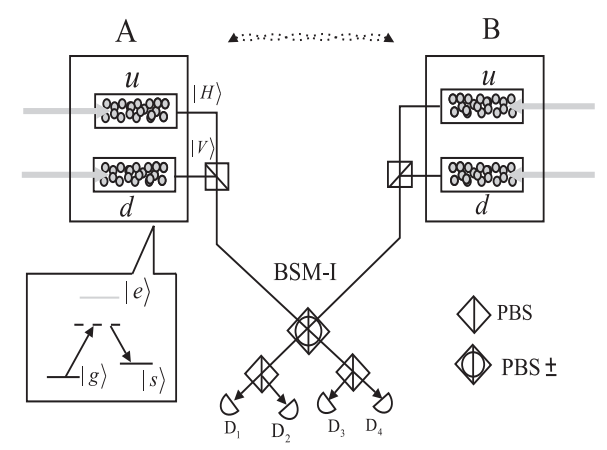

FIG. 1: Setup for entanglement generation between sites A and B. Forward-scattered Stokes photons, generated by an offresonant write laser pulse via spontaneous Raman transition, are subject to BSM-I at the middle point. The Stokes photons generated at the same site are assumed to have different polarization i.e., $|H\rangle$ and $|V\rangle$. PBS $\left(\mathrm{PBS}_{ \pm}\right)$reflects photons with polarization $|V\rangle(|-\rangle)$ and transmits photons with polarization $|H\rangle(|+\rangle)$, where $| \pm\rangle=\frac{1}{\sqrt{2}}(|H\rangle \pm|V\rangle)$. After passing through the $\mathrm{PBS}_{ \pm}$and PBS successively, the Stokes photons are detected by single photon detectors. A coincidence count between single photon detectors $\mathrm{D}_{1}$ and $\mathrm{D}_{4}\left(\mathrm{D}_{1}\right.$ and $\left.\mathrm{D}_{3}\right)$ or $D_{2}$ and $D_{3}\left(D_{2}\right.$ and $\left.D_{4}\right)$ will project the four atomic ensembles into the complex entangled state $|\psi\rangle_{A B}$ up to a local unitary transformation. The inset shows the atomic level structure, with the ground state $|g\rangle$, metastable state $|s\rangle$, and excited state $|e\rangle$.

by neglecting higher order terms, where $\left|0_{a}\right\rangle=\bigotimes_{i}|g\rangle_{i}$ is the ground state of the atomic ensemble and $\left|0_{s}\right\rangle$ denotes vacuum state of the Stokes photon. Here, $a^{\dagger}$ is the creation operator of the Stokes mode and the collective atomic excitation operator is defined by $S^{\dagger}=$ $\frac{1}{\sqrt{N}} \sum_{i}|s\rangle_{i}\langle g|$, where $|s\rangle$ is the metastable atomic state. The small excitation probability $\chi \ll 1$ can be achieved by manipulating the write laser pulse [14].

The entanglement generation setup is shown in Fig. 1. Let us consider two sites $\mathrm{A}$ and $\mathrm{B}$ at a distance of $L_{0} \leq L_{a t t}$, with $L_{a t t}$ the channel attenuation length. Each site has two atomic ensembles encoded as one memory qubit and the two atomic ensembles at each node are excited simultaneously by write laser pulses. We assume the Stokes photons generated from the two atomic ensembles at the same site have orthogonal polarization state, e.g., $|H\rangle$ and $|V\rangle$, which denote horizontal and vertical linear polarization respectively. In this way the memory qubit is effectively entangled with the polarization state of the emitted Stokes photon.

The Stokes photons generated from both the sites are directed to the polarization beam splitter (PBS) and subject to BSM-I at the middle point to entangle the two neighboring memory qubits. However, the two-photon state generated in the second-order Spontaneous Raman process will also induce a coincidence count on the detectors. Thus BSM-I can only prepare the neighboring mem-

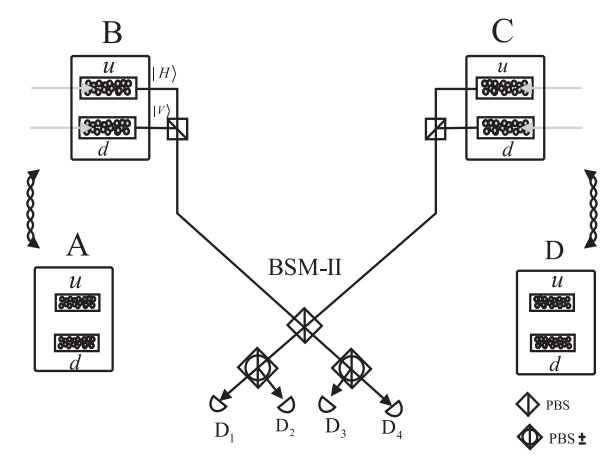

FIG. 2: Setup for entanglement connection between sites A and D via entanglement swapping. Complex entangled states have been prepared in the memory qubits between sites (A,B) and $(\mathrm{C}, \mathrm{D})$. The memory qubits at site $\mathrm{B}$ and $\mathrm{C}$ are illuminated by near resonant read laser pulses, and the retrieved antiStokes photons are subject to BSM-II at the middle point. The anti-Stokes photons at the same site have different polarizations $|H\rangle$ and $|V\rangle$. After passing through PBS and $\mathrm{PBS}_{ \pm}$ successively, the anti-Stokes photons are detected by single photon detectors. Coincidence count between $\mathrm{D}_{1}$ and $\mathrm{D}_{4}\left(\mathrm{D}_{1}\right.$ and $\left.D_{3}\right)$ or $D_{2}$ and $D_{3}\left(D_{2}\right.$ and $\left.D_{4}\right)$ are registered. The memory qubits will be projected into an effectively maximally entangled state $\rho_{A D}$ up to a local unitary transformation. Note that the PBS arrangement in BSM-II and BSM-I is different.

ory qubits into a complex superposition state with spurious contributions from second-order excitations. For instance, a coincidence count between $\mathrm{D}_{1}$ and $\mathrm{D}_{4}$ projects the two memory qubits into

$$
\begin{aligned}
|\psi\rangle_{A B}= & \frac{S_{u_{A}}^{\dagger} S_{u_{B}}^{\dagger}+S_{d_{A}}^{\dagger} S_{d_{B}}^{\dagger}}{2}+ \\
& \left.\frac{S_{u_{A}}^{\dagger 2}+S_{u_{B}}^{\dagger 2}-S_{d_{A}}^{\dagger 2}-S_{d_{B}}^{\dagger 2}}{4}\right)|v a c\rangle_{A B}
\end{aligned}
$$

by neglecting high-order terms, where the atomic ensembles are distinguished by subscript $(u, d)$ and $(A, B)$. The first part is the maximally entangled state needed for further operation, while the second part is the unwanted two-excitation state coming from second-order excitations. The success probability is on the order of $O\left(\chi^{2} \eta^{2} e^{-L_{0} / L_{a t t}}\right)$ by considering the channel attenuation, where $\eta$ is the detection efficiency. The time needed in this process is $T_{0} \approx \frac{T_{c c}}{\chi^{2} \eta^{2} e^{-L_{0} / L_{a t t}}}$, with $T_{c c}=L_{0} / c$ the classical communication time.

It is obvious the spurious contributions of twoexcitation terms prevent further entanglement manipulation and must be eliminated by some means. However we find that it is not necessary to worry about these terms, because they can be automatically washed out if the BSM in entanglement swapping is carefully designed. In ideal case a maximally entangled state can be created by implementing entanglement swapping.

The entanglement swapping setup is depicted in Fig. 2. Let us consider four communication sites $(A, B)$ and 
$(\mathrm{C}, \mathrm{D})$ and assume we have created complex entangled states $|\psi\rangle_{A B}$ and $|\psi\rangle_{C D}$ between sites (A,B) and (C,D) respectively. The memory qubits at site $\mathrm{B}$ and $\mathrm{C}$ are illuminated simultaneously by read laser pulses. The retrieved anti-Stokes photons are subject to BSM-II at the middle point between $\mathrm{B}$ and $\mathrm{C}$ (see Fig. 2). Note that the arrangement of the PBSs in BSM-II is carefully designed, so that the two-photon states converted from the unwanted two-excitation terms are directed into the same output and thus won't induce a coincidence count on the detectors. In ideal case, if the retrieve efficiency is unit and perfect photon detectors are used to distinguish photons' numbers, only the two-photon coincidence count will be registered and project the memory qubits into a maximally entangled state. For instance, when a coincidence count between $\mathrm{D}_{1}$ and $\mathrm{D}_{4}$ is registered one will obtain

$$
\left|\phi^{+}\right\rangle_{A D}=\left(S_{u_{A}}^{\dagger} S_{u_{D}}^{\dagger}+S_{d_{A}}^{\dagger} S_{d_{D}}^{\dagger}\right) / \sqrt{2}|v a c\rangle_{A D}
$$

In this way a maximally entangled state across sites A and $\mathrm{D}$ is generated by performing entanglement swapping.

However, for realistic atomic ensembles the retrieve efficiency $\eta_{r}$ is determined by optical depth of the atomic ensemble [15], and current single photon detectors are incapable of distinguishing photon numbers. Taking into account these imperfections, the multi-photon coincidence counts in BSM-II have to be considered. Through some simple calculations, one can find that the coincidence counts will prepare the memory qubits into a mixed entangled state of the form

$$
\rho_{A D}=p_{2} \rho_{2}+p_{1} \rho_{1}+p_{0} \rho_{0},
$$

where the coefficients $p_{2}, p_{1}$ and $p_{0}$ are determined by the retrieve efficiency and detection efficiency [16]. Here $\rho_{2}=\left|\phi^{+}\right\rangle_{A D}\left\langle\phi^{+}\right|$is a maximally entangled state, $\rho_{1}$ is a maximally mixed state where only one of the four atomic ensembles has one excitation and $\rho_{0}$ is the vacuum state that all the atomic ensembles are in the ground states.

It is easy to see that $\rho_{A D}$ is in fact an effectively maximally entangled states, which can be projected automatically to a maximally entangled state in the entanglement based quantum cryptography schemes. When we implement quantum cryptography via Ekert protocol 2], only the first term $\rho_{2}$ can contribute to a coincidence count between the detectors at the two sites and will be registered after classical communication. The maximally mixed state term $\rho_{1}$ and the vacuum term $\rho_{0}$ have no contribution to the experimental results, and thus $\rho_{A D}$ is equivalent to the Bell state $\left|\phi^{+}\right\rangle_{A D}=$ $\left(S_{u_{A}}^{\dagger} S_{u_{D}}^{\dagger}+S_{d_{A}}^{\dagger} S_{d_{D}}^{\dagger}\right) / \sqrt{2}|v a c\rangle_{A D}$.

The effectively entangled state can be connected to longer communication distance via further entanglement swapping. Taking into account higher-order excitations, the effectively mixed entangled pair reads $\rho^{\prime}=\rho+p_{h}^{\prime} \rho_{h}^{\prime}$,

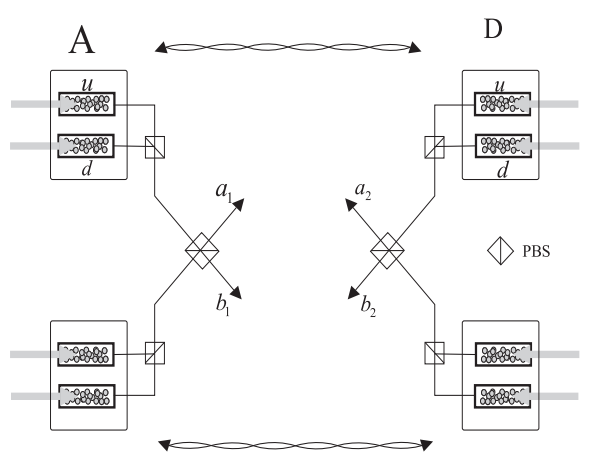

FIG. 3: Setup for quantum entanglement purification. Effectively entangled states have been prepared in the memory qubits between two distant sites $\mathrm{A}$ and $\mathrm{D}$. The memory qubits at the two sites are illuminated by near resonant read laser pulse, and the retrieved entangled photon pairs are directed to two PBS respectively. The photons in mode $b_{1}$ and $b_{2}$ are detected in $| \pm\rangle=\frac{1}{\sqrt{2}}(|H\rangle \pm|V\rangle)$ basis and the left photons in mode $a_{1}$ and $a_{2}$ are restored in the memory qubits at the two sites respectively.

where the normalized mixed state $\rho_{h}^{\prime}$ denotes contributions from higher-order excitations and the small coefficients $p_{h}^{\prime}$ is on the order of $O(\chi) \ll 1[16]$. After the $j$-th $(j \geq 2)$ swapping step, the effective entangled pair can be described as

$$
\rho_{s_{j}}^{\prime}=p_{2 s_{j}} \rho_{2 s_{j}}+p_{1 s_{j}} \rho_{1 s_{j}}+p_{0 s_{j}} \rho_{0 s_{j}}+p_{h s_{j}}^{\prime} \rho_{h s_{j}}^{\prime} .
$$

Here $\rho_{2 s_{j}}$ is the maximally entangled state between two memory qubits at a distance of $L=\left(2^{j+1}-1\right) L_{0}$, and $\rho_{1 s_{j}}, \rho_{0 s_{j}}$ are also the maximally mixed state and vacuum state respectively. Note that $\rho_{s_{1}}^{\prime}=\rho^{\prime}$ is just the mixed entangled state created after the first entanglement swapping step. The coefficients can be estimated to be [16]

$$
\begin{aligned}
& p_{h s_{j}}^{\prime} \sim O(j \chi) \\
& p_{\alpha s_{j}} \approx p_{\alpha s_{j-1}}+O(j \chi),(\alpha=0,1,2)
\end{aligned}
$$

It is readily to find that the contributions from higherorder excitations can be safely neglected, as long as the small excitation probability fulfills $j \chi \ll 1$, which can be easily achieved by tuning the write laser pulse. One can also see that the coefficients $p_{2 s_{j}}, p_{1 s_{j}}$ and $p_{0 s_{j}}$ are stable to the first order, therefore the probability to find an entangled pair in the remaining memory qubits is almost a constant and won't decrease significantly with distance during the entanglement connection process. The time needed for the $j$-th connection step satisfies the iteration formula $T_{s_{j}}=\frac{1}{p_{s_{j}}}\left[T_{s_{j-1}}+2^{j} T_{c c}\right]$, where the success probability $p_{s_{j}}$ is on the order of $O\left(\eta_{r}^{2} \eta^{2} e^{-L_{0} / L_{a t t}}\right)$. The total time needed for the entanglement connection process is

$$
T_{t o t} \approx T_{0} \prod_{j} p_{s_{j}}^{-1} \approx \frac{T_{c c}}{\chi^{2}} e^{L_{0} / L a t t}\left(L / L_{0}\right)^{\log _{2}^{1 / p}}
$$


where $p=\eta_{r}^{2} \eta^{2} e^{-L_{0} / L_{a t t}}$ is a constant. The excitation probability can be estimated to be $\chi \sim L_{0} / L$, and then the time needed in the entanglement connection process $T_{\text {tot }} \propto\left(L / L_{0}\right)^{2+\log _{2}^{1 / p}}$ scales polynomially or quadratically with the communication distance.

The effectively maximally entangled state generated above may be imperfect due to decoherence. For simplicity, assume a mixed state $\rho_{2}=F\left|\phi^{+}\right\rangle_{A D}\left\langle\phi^{+}\right|+(1-$ $F)\left|\psi^{+}\right\rangle_{A D}\left\langle\psi^{+}\right|$is created after entanglement swapping, where $\left|\psi^{+}\right\rangle_{A D}=\left(S_{u_{A}}^{\dagger} S_{d_{D}}^{\dagger}+S_{d_{A}}^{\dagger} S_{u_{D}}^{\dagger}\right) / \sqrt{2}|v a c\rangle_{A D}$. The mixed entangled state can be purified by linear-optics entanglement purification protocol [17]. As shown in Fig. 3, two effectively mixed entangled pairs are created in parallel via entanglement swapping. The effectively entangled states stored in the four memory qubits are converted into entangled photons by the read laser pulses, and then subject to two PBSs respectively. The photons in mode $b_{1}$ and $b_{2}$ are detected in $| \pm\rangle=\frac{1}{\sqrt{2}}(|H\rangle \pm|V\rangle)$ basis by single photon detectors, and will project the photons in mode $a_{1}$ and $a_{2}$ into an effectively maximally entangled state of higher fidelity $F^{\prime}=\frac{F^{2}}{F^{2}+(1-F)^{2}}$ [17]. The higherfidelity entangled pair in mode $a_{1}$ and $a_{2}$ can be restored into two distant memory qubits by means of dark-statepolariton [18] for further manipulation.

To generate a remote entangled pair, nested quantum purification has to be implemented. The total time overhead to create entanglement across two communication nodes at a distance of $1270 \mathrm{~km}$ can be numerically estimated. In our calculation, we assume the distance $L_{0}=10 \mathrm{~km}$ and thus the connection step $j=6$. The photon loss rate is considered to be $0.1 \mathrm{~dB} / \mathrm{km}$ in free space as reported [19]. The initial fidelity is assumed to be $F=0.88$. To increase the efficiency, we assume high efficiency (99\%) photon counting detectors based on atomic ensembles are used [20], and the retrieve efficiency is considered to be $98 \%$. Entanglement purification is performed twice during the whole process to improve the fidelity. Our numerical results give a total time of about three hours to create an effectively entangled pair, with a probability of 0.85 to get the entangled pair of fidelity $95 \%$ 16]. We note that the time overhead can be reduced significantly by optimization.

In our scheme, entanglement creation and swapping are both performed remotely, since they rely on twophoton interference. For the sake of scalability, entanglement generation could be locally performed, because it is usually rate-limiting stage due to the low excitation probability. The prize to pay is that one has to manipulate at least two memory qubits at each site. The locally entangled pair can also be generated by storing polarization entangled photons. Let us assume memory qubits A and B (see Fig. 1) are at one site and that we have created polarization entangled photon pair with the help of single photon source [21]. By sending the two entangled photons into memory qubits $\mathrm{A}$ and $\mathrm{B}$ respectively and storing them via dark-state-polariton, one can generate local entanglement between memory qubits A and B. Entanglement swapping and entanglement purification discussed above also apply to the entangled memory qubits and thus allow implementation of a robust quantum repeater. In this case, on demand single photon source will help to improve the scalability significantly [6].

In summary, we have proposed a robust and feasible quantum repeater by means of two photon Hong-OuMandel-type interference. Long-lived quantum memory is crucial for implementing the atomic ensemble based quantum repeater. In a recent proposal [22], it was shown that the ground state of ${ }^{3} \mathrm{He}$ has the potential to store a quantum state for times as long as hours.

This work was supported by the DFG, the NFRP and the European Commission.

Note added.- After the paper was finished, a similar work by L. Jiang et al. [23] appeared on arXiv.

[1] N. Gisin et al., Rev. Mod. Phys. 74, 145 (2002).

[2] A.K. Ekert, Phys. Rev. Lett. 67, 661 (1991).

[3] H.-J. Briegel et al., Phys. Rev. Lett. 81, 5932 (1998); W. Dür et al. Phys. Rev. A 59, 169 (1999).

[4] L.M. Duan et al., Nature(London) 414, 413 (2001).

[5] A. Kuzmich et al., Nature(London) 423, 731 (2003); C.H. van del Wal et al., Science 301, 196 (2003).

[6] D.N. Matsukevich et al., Phys. Rev. Lett. 97, 013601 (2006); S. Chen et al., ibid. 97, 173004 (2006).

[7] C.W. Chou et al., Nature(London) 438, 828 (2005).

[8] K.W. Holman, D.D. Hudson, and J. Ye, Opt. Lett. 30, 1225 (2005).

[9] M.D. Eisaman, et al., Nature(London) 438, 837 (2005).

[10] D. Felinto et al., Nature Physics 2, 844 (2006); T. Chanelière et al., Phys. Rev. Lett. 98, 113602 (2007); S.-Z. Yuan, et al., quant-ph/0703188.

[11] S. Bose et al., Phys. Rev. Lett. 83, 5158 (1999); D.E. Browne, M.B. Plenio and S.F. Huelga, ibid. 91, 067901 (2003); X.-L. Feng et al., ibid. 90, 217902 (2003); C. Simon and W.T.M. Irvine, ibid. 91, 110405 (2003).

[12] D.E. Browne and T. Rudolph, Phys. Rev. Lett. 95, 010501 (2005).

[13] D.N. Matsukevich and A. Kuzmich, Science 306, 663 (2004).

[14] A. André, PhD thesis, Harvard University (2005).

[15] A.V. Gorshkov et al., quant-ph/0604037 (2006).

[16] B. Zhao, et. al., to be published.

[17] J.-W. Pan et al., Nature(London) 410, 1067 (2001); J.W. Pan et al., ibid. 423, 417 (2003).

[18] M.D. Lukin, S.F. Yelin and M. Fleischhauer, Phys. Rev. Lett. 84, 4232 (2000); M. Fleischhauer and M.D. Lukin, ibid. 84, 5094 (2000).

[19] C.-Z. Peng et al., Phys. Rev. Lett., 94, 150501 (2005).

[20] D.F.V. James and P.G. Kwiat, Phys. Rev. Lett. 89, 183601 (2002); A. Imamoḡlu, ibid. 89, 163602 (2002).

[21] Q. Zhang et. al, quant-ph/0610145.

[22] A. Dantan et al., Phys. Rev. Lett. 95, 123002 (2005).

[23] L. Jiang et al., quant-ph/0609236. 